\title{
SMART FILTERING OF INTERFEROMETRIC PHASES FOR ENHANCING BUILDING RECONSTRUCTION
}

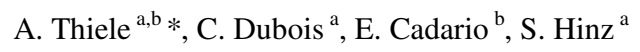 \\ ${ }^{a}$ Karlsruhe Institute of Technology (KIT), Institute of Photogrammetry and Remote Sensing (IPF), 76131 Karlsruhe, \\ Germany - (antje.thiele, stefan.hinz)@kit.edu, clemence.dubois@student.kit.edu \\ ${ }^{\mathrm{b}}$ Fraunhofer Institute of Optronics, System Technologies and Image Exploitation (IOSB), 76275 Ettlingen, \\ Germany - (antje.thiele, erich.cadario)@iosb.fraunhofer.de
}

Working Groups I/2, III/1, III/4, III/5

KEY WORDS: Remote Sensing, Building Reconstruction, High Resolution InSAR

\begin{abstract}
:
The current generation of space borne high resolution SAR sensors provides high spatial resolution as well as interferometric data within short time frames. This makes such data attractive for 3D information extraction. Especially, the operational configuration of TerraSAR-X and TanDEM-X opens up new perspectives for this kind of applications. Despite of this, the interferometric phases still suffer from considerable noise, so that filtering is mandatory to enhance building reconstruction.

In our previous work, we used conventional Multilook-filtering to smooth the phase signature. For large buildings acceptable filter results are shown, but signatures of small buildings and significant layover areas are destroyed by the use of large square windows. Such filters are especially inappropriate if building orientations are not aligned with the sensor flight direction. Hence, in this paper, we present modified InSAR phase filters to support 3D building reconstruction. The implementation focuses on two different strategies: on the one hand taking GIS information into account, in order to parameterize the filters accordingly, and on the other hand purely relying on the image data. The filters are tested on simulated interferometric phases and on real single-pass airborne InSAR data. Finally, filter properties are compared with current standard InSAR filters.
\end{abstract}

\section{INTRODUCTION}

\subsection{Motivation}

In the last years the new generation of spaceborne high resolution SAR sensors such as TerraSAR-X, SAR-Lupe, Cosmo-SkyMed or RADARSAT-2 provides SAR images of meter resolution or even better in special spotlight modes, which open up the floor for many new applications. In particular, the development of methods to automatically derive detailed cartographic information of both rural and urban areas from this kind of data is a major issue driven by these missions. Now, the newest SAR satellite sensor systems provide short repeat-pass or even highly coherent, single-pass interferometric data, which makes such data attractive for 3D information extraction. In particular the operational configuration of TerraSAR-X and TanDEM-X opens up new perspectives for this kind of applications.

Building detection from InSAR data presented in the literature was mainly based on a combined analysis of magnitude and interferometric height data (Bolter 2001, Soergel et al. 2003, and Thiele et al. 2007a). The utilization of the magnitude signature focused mainly on analyzing layover and shadow areas; and the analysis of the interferometric heights was mostly restricted to mean height calculation within an estimated building footprint. Yet, in high resolution InSAR data, even the shape of the interferometric phase profile at building locations contains valuable information (Thiele et al. 2007b).

A concept of exploiting this information for $3 \mathrm{D}$ building reconstruction was already presented in Thiele et al. 2010. Our reconstruction utilizes available 2D GIS information (building footprint) to simulate interferometric phase signatures of buildings. An iterative process of assessing real and simulated InSAR phases, of updating building model and of repeating simulation is set up to achieve best reconstruction results.

First tests revealed, that the assessment step between the simulated and real measured InSAR phases is the most crucial point. The reliability of the results depends on sensor configuration (e.g., baseline length), on quality of InSAR data (e.g., single-pass, repeat-pass), on used post-processing (e.g., filtering) and on matching between building model and real building. Hence, we now focus on the optimization of the smoothing of noisy InSAR phases using smart filtering.

Conventional Multilook-filtering (Lee et al. 1994) yields acceptable results when applied to large homogenous areas, but characteristic phase signatures, which appear also within layover areas of buildings, or signatures of smaller buildings were destroyed in particular by the use of large filtering windows. Similarly, such approaches are inappropriate if building orientations are not aligned with the sensor flight direction.

\subsection{Related Work}

InSAR filters like proposed in Goldstein et al. 1998 and Baran et al. 2003 investigate the frequency spectrum of an InSAR patch to reduce high frequency noise in the InSAR phases. Additionally, a weighting is applied depending on scene properties or local coherence values. Another filter approach presented in Tupin 2011 analyses coherence between so-called non-local image areas to de-noise image patches by a regularization-based method. This study is motivated by speckle reduction in SAR intensity data, but promising results are also shown on InSAR phase data.

\footnotetext{
* Corresponding author.
} 

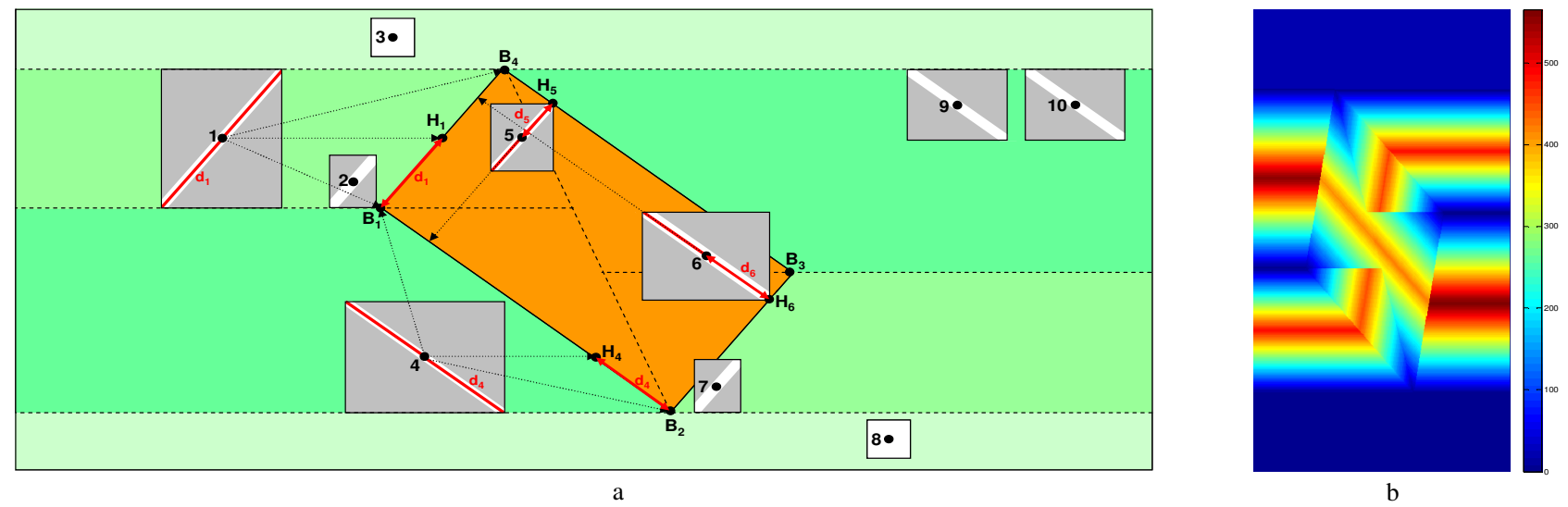

Figure 1. Adaptive phase filtering: a) schema of filter approach and b) 2D histogram of adaptive filter size [pixel number]

Improvements on conventional Multilook-filtering are described in Reeves et al. 1999 to increase smoothing while avoiding the elimination of significant local features. For this reason interferometric amplitudes are used to specify filter weights, which are finally determined by utilizing a MonteCarlo scheme. Approaches on adaptive complex Multilooking motivated by coherence filtering are published by Ciuc et al. 2002 and Vasile et al. 2004. Both start with a statistical analysis of SAR amplitude data, followed by a twostage region growing on the same data. Subsequently, a complex averaging in the segmented adaptive neighbourhood is achieved by considering different weighting functions.

A windowing and segmentation independent approach on phase noise modelling and reduction is described in LópezMartínez et al. 2002. The filtering of phase noise is accomplished by a local analysis in the wavelet domain, which enables high computational efficiency. Phase filtering by investigating morphological operators was presented in Rejichi et al. 2010. Based on gradient estimation in interferometric phases an alternate sequence of opening and closing operators is applied. Lee et al. 1998 implemented an adaptive orientation filter based on local noise level in the phase data. Sixteen orientations are considered and the local weighting is characterized by locale coherence, number of looks, and locale variance. A related approach is given in Bo et al. 1999 by extending the number of orientation masks. Two different values of line thickness are defined to preserve small signature details. The filtering and weighting is characterized by two options median or mean, similar to Lee et al. 1998.

Our implementations focus on two different strategies: on the one hand taking GIS information into account, and on the other hand, relying purely on image data without using additional GIS information. The paper is organized as follows: In Sect. 2, we outline the modifications and development for our smart filtering schemes. Test data are introduced in Sect. 3, followed by the visual and numerical validation of the filter results in Sect. 4. Conclusion and outlook are eventually given in Sect. 5.

\section{INTERFEROMETRIC PHASE FILTERING}

The investigated filters as well our adapted and new filters can be subdivided into three different groups characterized by the chosen type of filter window and by the introduction of prior information.

The first group contains the patch filter applied in the frequency domain on complex data (Goldstein-filter and Baran-filter) or in the spatial domain on phase data (coherence weighted Meanfilter and Median-filter).

The second group utilizes filter masks of different orientation to enable an adaptive averaging depending on local noise level or additional information. An adapted Lee-filter (org. Lee-filter without local unwrapping) and GIS-information (only orientation) related fixed-window-filter (GIS FWF) are considered.

The third group - adaptive-window filters - integrate building footprints. For these GIS-information related adaptive-windowfilters (GIS AWF), coherence weighted averaging (mean+coherence) and median searching (median) are implemented. The parameterization of the adaptive filter window is visualised in Figure 1a. Considering a single building, the surrounding and the building (corner points $\mathrm{B}_{1}, \mathrm{~B}_{2}, \mathrm{~B}_{3}, \mathrm{~B}_{4}$ ) are divided in three zones (dashed lines and different green colours), where different adaptive filter windows are applied. For the first area containing the example point 3 and 8 only a $5 \times 5$ square window is used. Exemplary filter windows for the second zone are given at the points $1,2,5,7$ and for the third at points $4,6,9,10$. The respective orientation in the filter window (considered pixels white marked) depends on the orientation of corresponding (closest) building side. The length of filter diagonals of pixels outside building footprint is defined by the doubled distance between $\mathrm{H}_{\mathrm{i}}$ and the nearest building corner (see $d_{1}$ and $d_{4}$ ). For pixels inside the building, the perpendicular distance to the opposite building side is used (see $\mathrm{d}_{5}$ and $\mathrm{d}_{6}$ ). In addition, a map indicating the number of pixels taken into account for averaging and median search is given in Figure $1 b$.

The above filtering schemes are applied to the wrapped phases. Future work will include the implementation of local phase unwrapping during filtering. One solution is the calculation of a mean phase level in a neighbourhood like Lee et al. 1998. For this approach, an additional integration of prior information (e.g., simulated InSAR phase) might be even more useful.

\section{TEST DATA}

The different groups of filters are applied on simulated data and real InSAR data sets showing the same industrial building with a size of approx. $60 \mathrm{~m}$ width and approx. $100 \mathrm{~m}$ length. The investigation on simulated phase data allows to investigating the different filtering behaviour in detail, especially for significant signature changes, e.g., between layover area and building roof area. Due to the fact that the utilized phase simulation approach delivers no complex data but only phases, some current phase filters can only be tested on real InSAR data. To this end, single-pass airborne SAR imagery was used. 


\subsection{Simulated Interferometric Phase Data}

For the simulation of interferometric phase, we use an adapted version of the approach presented in (Thiele et al. 2007b). A 3D building model and some SAR sensor parameters are needed as input. The building footprint is expected to be available from GIS data (Figure 2a,b) and the sensor parameters are chosen similar to the investigated real InSAR data. In contrast to earlier work, we compute not only a single phase profile per building hypothesis is calculated but the full interferometric phase signature of the building. In the simulation step we consider the fact that, especially at building locations, a mixture of several scattering effects can contribute to the measured interferometric phase within a single resolution cell. Hence, phases in the layover area show the so-called front porch shape mentioned in the literature. An example of the simulation result is given in Figure 2c.

For testing the different filter approaches, the simulated phases have to be corrupted by noise. The well-known noise distributions of the following instances are used contributing to the noisy (simulated) phase $\Delta \varphi_{\text {noisy }}$ :

$\Delta \varphi_{\text {noisy }}=\Delta \varphi_{\text {sim }}+\Delta \varphi_{\text {thermal }}+\Delta \varphi_{\text {coberent }}+\Delta \varphi_{\text {shadow }}$

where $\Delta \varphi_{\text {sim }}=$ simulated phases

$\Delta \varphi_{\text {thermal }}=$ thermal noise (normal distribution)

$\Delta \varphi_{\text {coberent }}=$ coherent noise (normal distribution)

$\Delta \varphi_{\text {shadow }}=$ shadow noise (uniform distribution, $[-\pi, \pi]$ ).
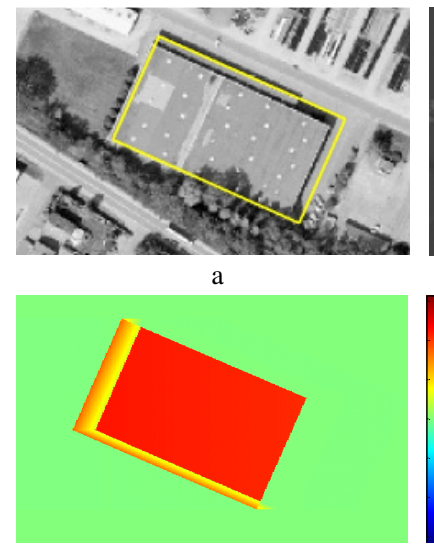

c

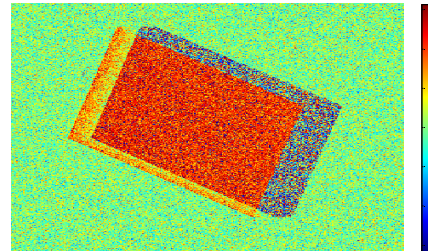

e

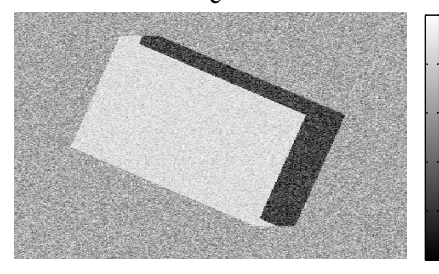

$\mathrm{g}$

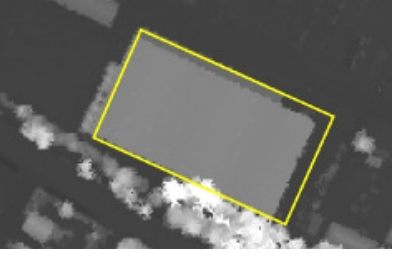

b
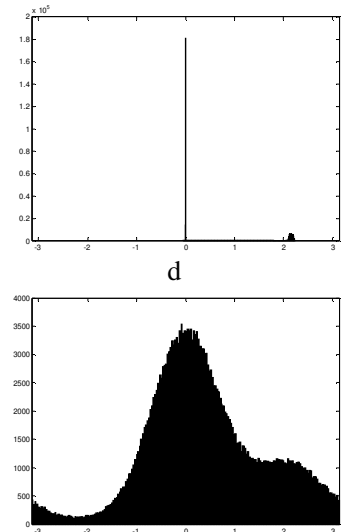

$\mathrm{f}$

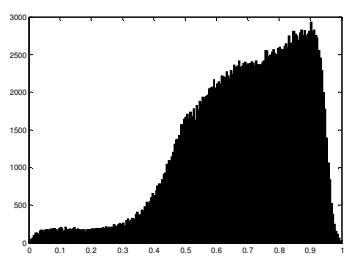

$\mathrm{h}$
Figure 2. Simulated building signature: a) optical and b) LIDAR image overlaid with GIS information, c) simulated phases and d) histogram, e) noisy simulated phases and f) histogram, g) noisy simulated coherence and h) histogram
The resulting noisy simulated phases are given in Figure 2e with the corresponding histogram in Figure 2f. Additionally, the simulation of a corresponding coherence map was on demand to enable more filter tests on the noisy simulated phases. The

$\operatorname{coh}_{\text {noisy }}=\operatorname{coh}_{\text {ground }}+\operatorname{coh}_{\text {building }}+\operatorname{coh}_{\text {shadow }}+\operatorname{coh} h_{\Delta \varphi}$

where $c o h_{\text {ground }}, c o h_{\text {building }}, c o h_{\text {shadow }}$ are formed by the normal distribution (ndf) of different $\mu, \sigma$ combinations, corresponding to measurements in real InSAR data. The last component $c_{0 h}$ is added to make a connection to noisy simulated phase in pixel space. The resulting noisy coherence map is given in Figure $2 g$ with the corresponding histogram in Figure $2 \mathrm{~h}$.

\subsection{Real Interferometric SAR Data}

The choice of real InSAR data was driven by minimizing noise due to temporal decorrelation, since this kind of noise can be almost neglected for satellite systems like TanDEM-X. Hence, we performed our tests with imagery of the airborne sensor Aes1 (Schwäbisch et al. 1999). The system operated in X-band at $3000 \mathrm{~m}$ flight height with a spatial resolution of about $38 \mathrm{~cm}$ in range and $17 \mathrm{~cm}$ in azimuth direction. The baseline was about $2.4 \mathrm{~m}$ and the scene was illuminated with an off-nadir angle spanning a range from $28^{\circ}$ up to $52^{\circ}$.

In Figure 3, the InSAR signature of the industrial building is shown. The direct visual comparison of simulated and real InSAR phase and coherence signatures shows high correlations. Differences in the phase signatures are only visible in the layover area due to occlusion effects caused by closed trees. Coherence values of real InSAR data at the building roof are in some cases lower due to different backscatter properties. Differences in histogram shape are caused by areas less decorrelated (e.g., streets, tree shadows) in the simulated InSAR phases.
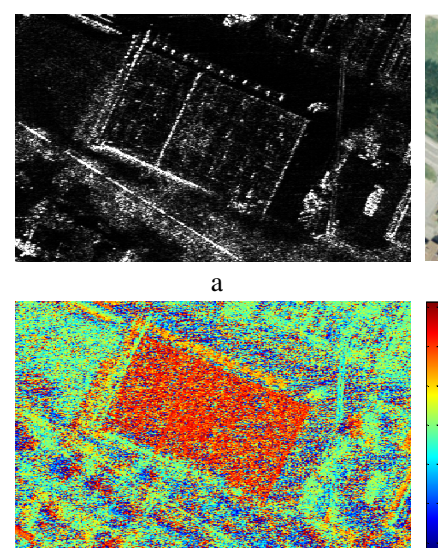

$\mathrm{c}$

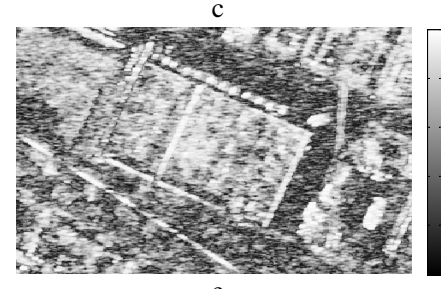

$\mathrm{e}$

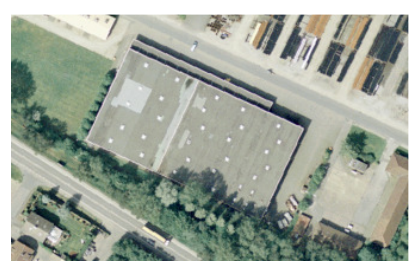

b

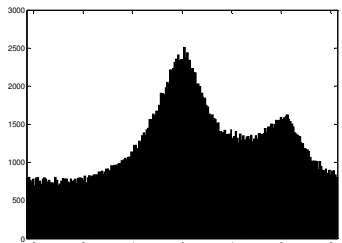

d

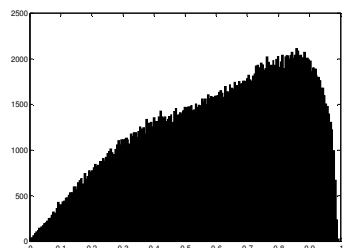

Figure 3. Real building signature: a) SAR magnitude, b) optical image, c) real phases and d) histogram,

e) real coherence and f) histogram 

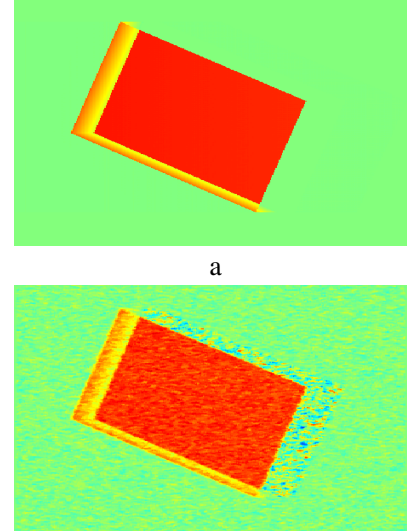

$\mathrm{c}$
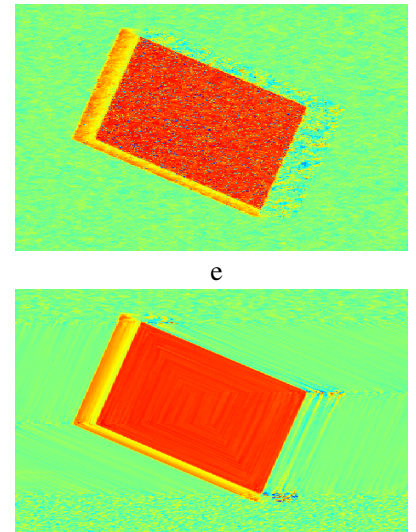

$\mathrm{g}$

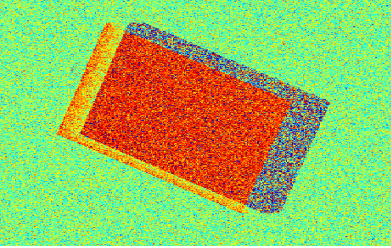

b

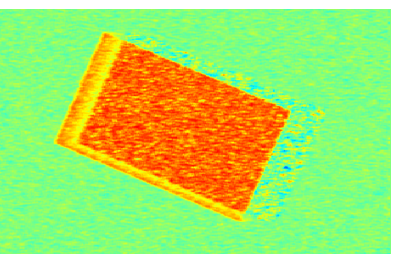

d

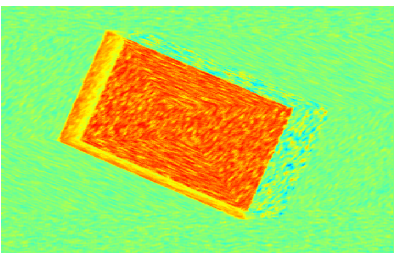

f

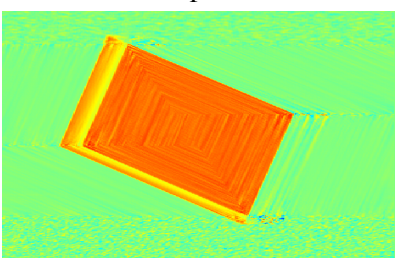

h

Figure 4. Filtering of simulated interferometric phases: a) original simulation, b) noisy simulation, c) median-filter, d)

mean-coherence-filter, e) adapted Lee-filter, using GIS

information for $\mathrm{f}$ ) fixed-window-filter (mean-coherence), g) adaptive-window-filter (median), and h) adaptive-window-filter (mean-coherence)

\section{VALIDATION OF FILTERING RESULTS}

The validation of filter results comprises a visual comparison of filtered building phase signatures and their differences to noisefree simulated phases, as well as a numerical assessment (mean local standard deviation of the filtered phases, variance of phase differences, correlation level between filtered and noisefree simulated phases). For the majority of filters we used a $5 \mathrm{x} 5$ pixel filter window to achieve comparable results.
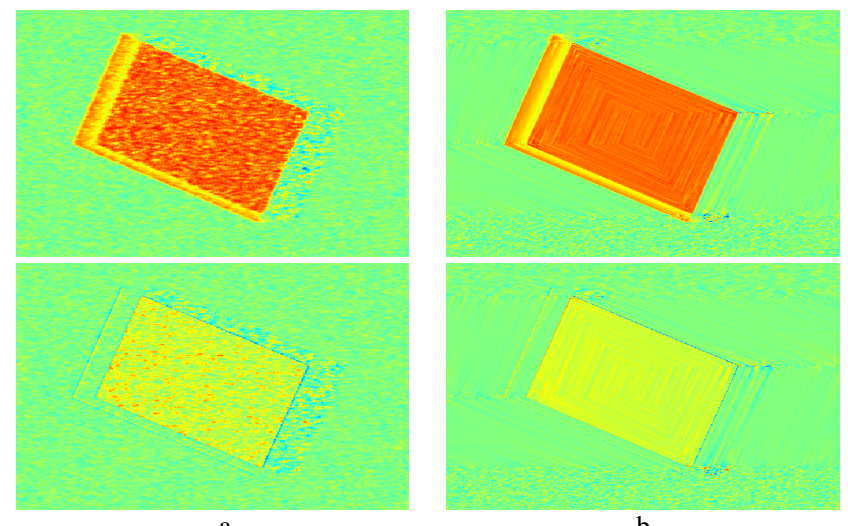

b

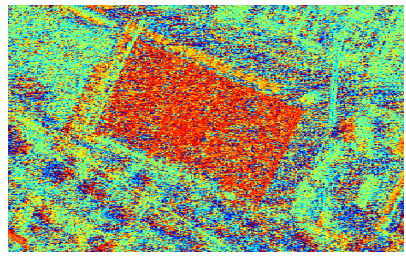

a

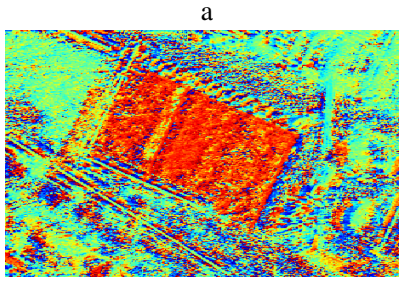

c

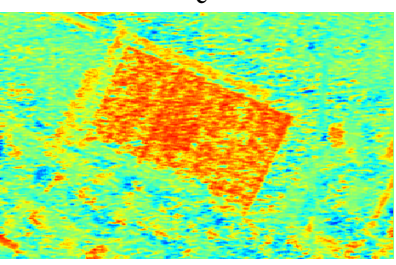

e

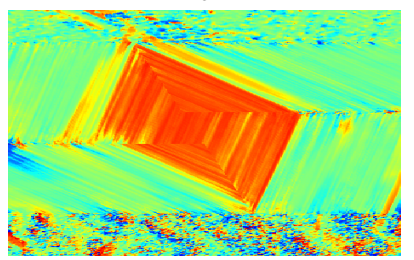

$\mathrm{g}$

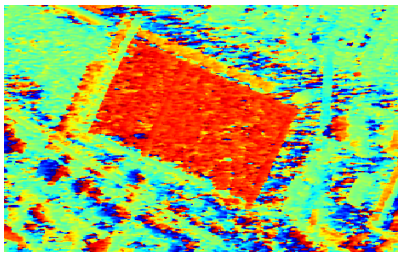

b

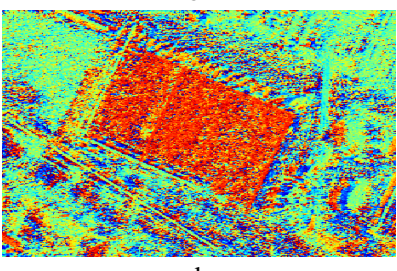

d

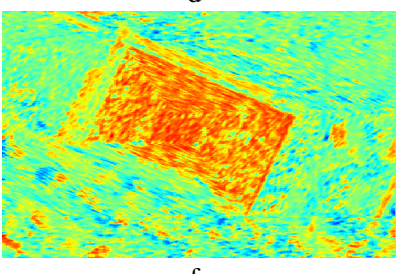

$\mathrm{f}$

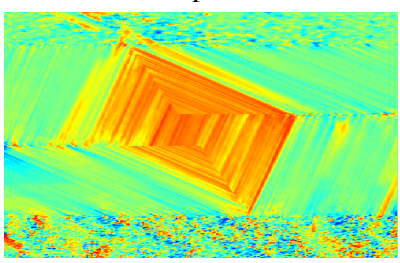

$\mathrm{h}$

Figure 5. Filtering of real interferometric phases: a) original measured interferometric phases, b) Multilook-filter, c)

Goldstein-filter, d) Baran-filter, e) mean-coherence-filter, using GIS information for f) fixed-window-filter (mean-coherence), g) adaptive-window-filter (median), and h) adaptive-window-filter (mean-coherence)

\subsection{Visual Validation}

The filter results on simulated and on real InSAR data are summarized in Figure 4 and 5. The best filter potential concerning phase-based building reconstruction is visible for the new implemented GIS AWFs (Figure 4g,h and Figure 5g,h) as it preserves the shape of the layover area best. Furthermore, tests on the new area filter (mean-coherence, Figure 4d, 5e) and GIS supported FWF show also promising results. Nevertheless, focussing on roof substructures the adaptive filtering can lead to
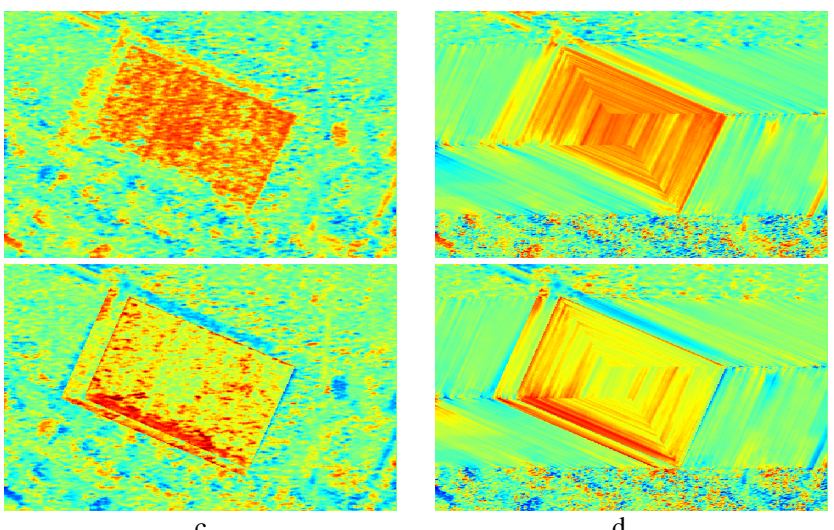

d

Figure 6. Filter results and differences between of noise-free simulated phases (Figure 4a) and filtered interferometric phases: a) Mean-coherence-filter and b) GIS AWF (mean+coh) on noisy simulated phases, c) Mean-coherence-filter and d) GIS AWF $($ mean $+\mathrm{coh})$ on real interferometric phases 


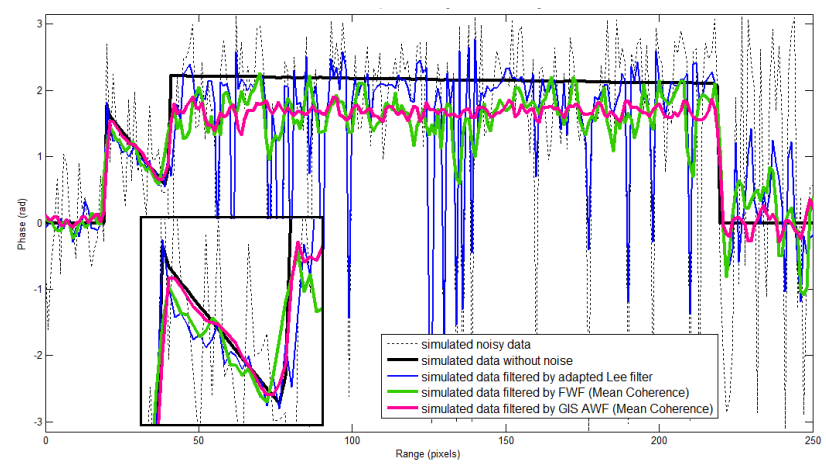

a

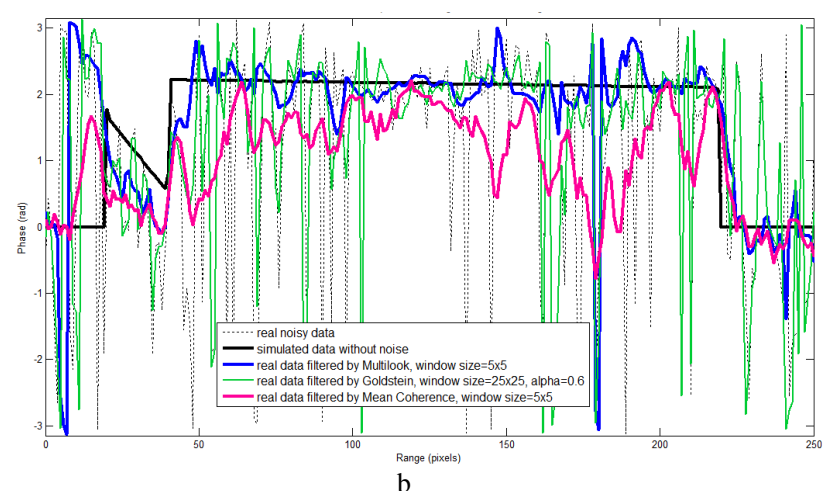

b

Figure 7. Slant-Range Profiles: a) filtering of noisy simulated phases and b) filtering of real interferometric phases

blur effects, which can be an advantage or disadvantage depending on application.

In Figure 6, phase plots showing difference between filtered and noise-free simulate phases are presented. The difference maps yield some border effects and a bias at the building roof. The first effect engendered by averaging and chosen filter width is acceptable; the second - probably caused by the missing phase unwrapping - have to be further investigated in future. The differences between real and simulated data (Figure 6c,d) stress the differences between assumed 3D building model and actual situation. In the real data, characteristic layover areas as well as a part of the roof are not visible due to neighboured trees (dark red in the difference map). Furthermore, the considered 3D building model does not contain a building substructure (e.g., canopy) marked blue in the upper part of the building. Hence, the matching of 3D building model and real building essentially affects the filtering result. Additionally, the registration of filter window and real building orientation has to be really good, which depends on the quality of GIS data and illumination / sensor parameters.

A more detailed comparison by focusing on preservation of layover shape and building roof level is possible on the profiles given in Figure 7, whereby a) summarizes the orientation filters and b) the area filters. Best results based on noisy simulated phases are achieved by the GIS AWF (mean+coh). The layover shape is preserved (see zoom-in at Figure 7a), noise is reduced, and only the mean phase level at building roof shows a bias since no local phase unwrapping is considered during filtering. Further investigations are planned at this point.

In the given example, the Multilook-filter shows best results in the group of area filters. The mean roof level is well-preserved and phase unwrapping is not necessary. Hence a combination of the two bests could be improving the filter results once again.

\begin{tabular}{|c|c|c|c|}
\hline Filter Name & $\begin{array}{c}\text { Standard } \\
\text { Deviation }\end{array}$ & Variance & $\begin{array}{c}\text { Cross } \\
\text { Correlation }\end{array}$ \\
\hline noisy simulated phase & 0.893 & 1.015 & 0.568 \\
\hline Median-filter & 0.157 & 0.062 & 0.958 \\
\hline Mean-Coherence-filter & 0.143 & 0.097 & 0.949 \\
\hline Adapted Lee-filter & 0.287 & 0.224 & 0.850 \\
\hline GIS FWF (mean+coh) & 0.155 & 0.094 & 0.951 \\
\hline GIS AWF (median) & 0.136 & 0.044 & 0.971 \\
\hline GIS AWF (mean+coh) & 0.120 & 0.075 & 0.969 \\
\hline
\end{tabular}

Table 1. Results of filtering based on simulated phases

\subsection{Numerical Validation}

The numerical validation of our filter results is based on three different values: the mean local standard deviation of filtered phases, the variance of differences (see Figure 6) and the cross correlation between filtered and noise-free simulated phases. The mean local standard deviation of the resulting interferometric phases $\overline{\sigma_{\Delta \varphi}}$ is defined by:

$\overline{\sigma_{\Delta \varphi}}=\frac{\sum_{i=1}^{m}\left(\frac{1}{n-1} \sum_{j=1}^{n}\left(\Delta \varphi_{j}-\overline{\Delta \varphi}\right)^{2}\right)^{1 / 2}}{m}$

where $n=25$, a local window of $5 \times 5$ pixel and $m$ contains the full image size.

The numerical results are summarized for noisy simulated phases in Table 1 and for real InSAR data in Table 2. Different filters are applied depending on provided data layer. Values given in brackets are only related to building layover and roof area - no ground and shadow area is considered. From the group of area filters shows the modified Median and MeanCoherence filter best results. An integration of a local phase unwrapping can probably especially improve the results of the Mean-Coherence filter again. For the group of orientation filters with fixed window size (adapted Lee-filter and GIS FWF) an improvement by investigating building orientation from GIS is visible. Results of the adaptive window size filters are similar to the area filter. The AWF Median show slightly better results than the AWF Mean-Coherence implementation. Furthermore, filter results on simulated data show a really high improvement, which is not comparable to tests on real InSAR data. Nevertheless, the high potential on appropriate phase filtering is demonstrated. The benefit of GIS AWFs compared to area filters will turns out more clearly by investigating data showing smaller building, which is planned in future publications.

\begin{tabular}{|c|c|c|c|}
\hline Filter Name & $\begin{array}{c}\text { Standard } \\
\text { Deviation }\end{array}$ & Variance & $\begin{array}{c}\text { Cross } \\
\text { Correlation }\end{array}$ \\
\hline Original phase data & 1.365 & $2.550(3.595)$ & $0.275(0.186)$ \\
\hline Multilook-filter & 0.547 & $1.352(0.975)$ & $0.525(0.496)$ \\
\hline Goldstein-filter & 1.081 & $2.182(2.470)$ & $0.375(0.307)$ \\
\hline Baran-filter & 1.1758 & $2.270(2.790)$ & $0.349(0.267)$ \\
\hline Mean-Coherence-filter & 0.287 & $0.548(1.230)$ & $0.628(0.471)$ \\
\hline GIS FWF (mean+coh) & 0.291 & $0.530(1.204)$ & $0.639(0.483)$ \\
\hline GIS AWF (median) & 0.300 & $0.534(0.662)$ & $0.681(0.621)$ \\
\hline GIS AWF (mean+coh) & 0.241 & $0.475(1.007)$ & $0.674(0.587)$ \\
\hline
\end{tabular}

Table 2. Results of filtering based on real InSAR phases 


\section{CONCLUSION AND OUTLOOK}

In this paper, we presented a modified and enhanced filter approaches to smooth interferometric phase signatures of buildings by incorporating available GIS information. Based on the relative pixel position an appropriate window size and window orientation of the phase filter is chosen. First results were shown on simulated and real measured InSAR phases of a single-pass airborne SAR sensor. The visual and numerical interpretation of the new filter result was supported by applying current filter (e.g., Multilook, Goldstein, Lee), too. These first results show a high potential of such GIS-driven filters, especially for applications of building reconstruction.

Our further steps will focus on additional tests considering different airborne and spaceborne data of single-pass and repeat-pass configurations (e.g, TerraSAR-X and TanDEM-X). Furthermore, the abovementioned local unwrapping problem during the phase filtering will be considered. The given solution in Lee et al. 1998 has to be compared with the information given by the phase simulation. Beside, improvements on Goldstein-filter and Baran-filter are planned, as well as the implementation of a combined adaptive GIS and area filter to enable reasonable adjustments to layover and roof areas. Further, investigations on building neighbourhood should be mentioned here, because interaction effects between buildings can necessitate the adaptation of phase filtering.

Finally, the overall goal will be the integration in the superordinate approach of automatic 3D building reconstruction utilizing GIS and mono- or multi-temporal InSAR data to support the following two applications:

- the analysis of mono-temporal TanDEM-X scenes to extract object information for supporting the planning of energy supply in the future, whereby building specific volumes have to be estimated for the evaluation of the energy density. Such data are used to generate building-based thermal maps, which provide another level of planning guides to support the setup of new innovative $\mathrm{CO} 2$-reduced heat combined systems.

- the analysis of multi-temporal TanDEM-X scenes. It is targeted to generate automatic comparison between the reconstruction $3 \mathrm{D}$ building structures and their new multitemporal InSAR signature. In case of important changes due to earthquakes or other natural disasters, the identification of changes is an appropriate support for rescuers.

\section{REFERENCES}

Baran, I., Stewart, M. P., Kampes, B. M., Perski, Z., Lilly P., 2003 A Modification to the Goldstein Radar Interferogram Filter. IEEE Transaction on Geoscience and Remote Sensing, vol. 41, no. 9, pp. 2114-2118.

Bo, G., Dellepiane, S., Beneventano, G., 1999 A locally adaptive approach for interferometric phase noise reduction. IEEE International Geoscience and Remote Sensing Symposium (IGARSS), doi: 10.1109/IGARSS.1999.773466, vol. 1, pp. 264-266.

Bolter, R., 2001 Buildings from SAR: Detection and Reconstruction of Buildings from Multiple View High Resolution Interferometric SAR Data. University Graz: Ph. D. Thesis.

Ciuc, M., Trouve, E., Bolon, P., Buzuloiu, V., 2002 Amplitude-driven coherence filtering in complex interferograms. IEEE International Geoscience and Remote
Sensing
Symposium
(IGARSS),
doi: 10.1109/IGARSS.2002.1027213, vol. 6, pp. 3453-3455.

Goldstein, R. M., Werner C. L., 1998 Radar interferogram filtering for geophysical applications. Geophysical Research Letters, vol. 25, no. 21, pp. 4035-4038.

Lee, J.-S., Hoppel, K. W., Mango, S. A., Miller A. R., 1994 Intensity and Phase Statistics of Multilook Polarimetric and Interferometric SAR Imagery. IEEE Transaction on Geoscience and Remote Sensing, vol. 32, no. 5, pp. 1017-1028.

Lee, J.-S., Papathanassiou, K. P., Ainsworth, T. L., Grunes, M. R., Reigber, A., 1998 A new technique for noise filtering of SAR interferometric phase images. IEEE Transaction on Geoscience and Remote Sensing, vol. 36, no. 5, pp. 1456-1465.

López-Martínez, C., Fàbregas, and X., 2002 Modeling and reduction of SAR interferometric phase noise in the wavelet domain. IEEE Transaction on Geoscience and Remote Sensing, vol. 40 , no. 12 , pp. $2553-2566$.

Reeves, B., Homer, J., Stickley, G., Noon, D., Longstaff, I.D., 1999 Spatial vector filtering to reduce noise in interferometric phase images. IEEE International Geoscience and Remote Sensing Symposium (IGARSS), doi: 10.1109/IGARSS.1999.773465, vol. 1, pp. 260-263.

Rejichi, S., Chaabane, F., Tupin, F., Bloch, I., 2010 Morphological filtering of SAR interferometric images. IEEE International Geoscience and Remote Sensing Symposium (IGARSS), doi: 10.1109/IGARSS.2010.5653767, pp. 15811584.

Schwaebisch, M., Moreira, J., 1999 The high resolution airborne interferometric SAR AeS-1. Proceedings of the Fourth International Airborne Remote Sensing Conference and Exhibition, Canada, pp. 540-547

Soergel, U., Thoennessen, U., Stilla, U., 2003 Iterative Building Reconstruction in Multi-Aspect InSAR Data. In: Maas HG, Vosselman G, Streilein A (eds) 3-D Reconstruction from Air-borne Laser-scanner and InSAR Data, IntArchPhRS, vol. 34, part 3/W13, pp. 186-192.

Thiele, A., Cadario, E., Schulz, K., Thoennessen, U., Soergel, U., 2007a Building recognition from multi-aspect high resolution InSAR data in urban area. IEEE Transactions on Geoscience and Remote Sensing, vol. 45, no. 11, pp. 35833593.

Thiele, A., Cadario, E., Schulz, K., Thoennessen, U., Soergel, U., 2007b InSAR Phase Profiles at Building Locations. Proceeding of ISPRS Photogrammetric Image Analysis, vol. XXXVI, part 3/W49A, pp. 203-208.

Thiele, A., Hinz, S., Cadario, E., 2010 Fusion of InSAR and GIS data for 3D building reconstruction and change detection. Proceedings of 'Fringe 2009 Workshop', Frascati, Italy, ESA SP-677, http://earth.eo.esa.int/workshops/fringe09/.

Tupin, F., 2011 How Advanced Image Processing Helps For SAR Image Restoration and Analysis. IEEE Geoscience and Remote Sensing Newsletter, Cumulative Issue 158, March 2011, ISSN: 0274-6338, pp. 10-17.

Vasile, G., Emmanuel, T., 2004 General adaptiveneighborhood technique for improving synthetic aperture radar interferometric coherence estimation. Journal of the Optical Society of America A, vol. 21, no. 8, pp. 1455-1464. 